\title{
Qualidade de painéis OSB (Oriented Strand Board) produzidos com a madeira de clones de Eucalyptus urophylla
}

\author{
José Benedito Guimarães Júnior ${ }^{1 \star}$, Rafael Farinassi Mendes ${ }^{2}$, Ingrid Luz Guimarães ${ }^{2}$, \\ Fernado Noraga Lisboa ${ }^{2}$, Thiago de Paula Protásio² ${ }^{2}$ Lourival Marin Mendes ${ }^{2}$ \\ ${ }^{1}$ Engenharia de Materiais, Universidade Federal de Lavras (UFLA), Lavras, Brasil. \\ ${ }^{2}$ Engenharia Florestal, Universidade Federal de Lavras (UFLA), Lavras, Brasil.
}

\begin{abstract}
RESUMO Esse trabalho teve como objetivo avaliar características físicas e mecânicas de painéis OSB (Oriented Strand Board) produzidos a partir de seis clones de Eucalyptus urophylla. Foram utilizadas partículas do tipo "strand" na proporção de 90\% em relação à massa seca do painel, encoladas com $9 \%$ do adesivo fenol-formaldeído e $1 \%$ de emulsão de parafina. Os painéis foram prensados por 10 minutos a uma temperatura de $150^{\circ} \mathrm{C}$, pressão de $3,92 \mathrm{MPa}$ e tiveram densidade nominal de $0,70 \mathrm{~g} / \mathrm{cm}^{3}$. Posteriormente, foram avaliados em suas propriedades físicas e mecânicas. Os painéis produzidos a partir dos clones 26 e 62 foram os que absorveram mais água em 2 horas de imersão. Quando avaliada em 24 horas de imersão, não houve diferença entre valores de absorção de água. Para a propriedade de inchamento em espessura, em 2 e 24 horas de imersão em água, os maiores valores foram observados para os painéis provenientes dos clones 62 e 28 , respectivamente. Todos os painéis apresentaram valores estatisticamente iguais para todas as propriedades mecânicas, com exceção do clone 19, que teve valores superiores aos demais para o MOE e MOR no sentido paralelo. De maneira geral, houve baixo efeito clonal nas propriedades dos painéis OSB.
\end{abstract}

Palavras-chave: partículas orientadas, eucalipto, fenol-formaldeído.

\section{Quality of OSB (Oriented Strand Board) panels produced with wood from Eucalyptus urophylla clones}

\begin{abstract}
The objective of this work was to evaluate the physical and mechanical properties of OSB (Oriented Strand Board) produced from six different clones Eucalyptus urophylla clones Particles "Strands" were used in the proportion of $90 \%$ of the relative dry mass of the panel, glued with $9 \%$ phenol-formaldehyde adhesive and $1 \%$ paraffin emulsion. The OSB panels were pressed for 10 minutes at $150^{\circ} \mathrm{C}$ temperature, $3.92 \mathrm{MPa}$ pressure and $0.70 \mathrm{~g} / \mathrm{cm}^{3}$ nominal density. After that, the panels had their physical and mechanical properties evaluated. The panels produced with clones 26 and 62 obtained the highest water absorption after two hours of immersion. When evaluated in 24 hours of immersion in water, there was no difference between water absorption values. For thickness swelling property, for 2 and 24 hours of immersion in water, the highest values were observed for the OSB panels produced from the clones 62 and 28, respectively. All OSB panels showed statistically equal values for all mechanical properties, excepting for clone 19, which had higher values than the other for MOE and MOR in the parallel direction. In general, there was a low clone effect on the properties of the OSB panels.
\end{abstract}

Keywords: oriented particles, eucalypt, phenol-formaldehyde.

\section{Introdução}

Os painéis de madeira reconstituída podem ser uma alternativa ao uso da madeira maciça, principalmente como elementos estruturais, embalagens, indústria moveleira, dentre outros (BERTOLINI et al., 2013).
No Brasil, o setor de painéis tem apresentado grande crescimento devido à modernização do parque fabril e o surgimento de novos produtos, além do aumento da demanda dos 
setores de construção civil e de móveis, os quais são os principais consumidores de painéis de madeira (MENDES et al., 2013).

Dentre esses painéis, destaca-se o OSB, que de acordo com Maloney (1993), é um painel de partículas de madeira orientadas (strands), coladas com adesivo à prova d'água e prensadas a quente. As partículas da camada interna podem estar dispostas aleatoriamente ou perpendicularmente em relação às camadas externas. Esta conformação confere melhor distribuição da resistência nas direções longitudinal e transversal, além de melhorar a estabilidade dimensional.

Este produto está no mercado mundial desde o início da década de 1980, sendo considerado como a segunda geração dos painéis Waferboard. No Brasil, a produção de OSB ainda é muito incipiente, começando a produzir painéis somente no ano de 2002 (GOSKI et al., 2015).

As chapas OSB são produtos utilizados para aplicações estruturais, como paredes, suportes para forros e pisos, componentes de vigas estruturais, estrutura de móveis, embalagens, entre outras, tendo em vista boas características de resistência mecânica e estabilidade dimensional (MARTARELLO et al., 2015)

No que se refere a matéria-prima, Castro (2012) afirma que a produção nacional de OSB utiliza predominantemente madeira de Pinus spp. No entanto, com a crescente demanda desse produto e, sendo esta madeira utilizada para diversas outras finalidades, se torna necessária a busca por novas espécies que possam se adequar ao processo.

As variações existentes entre espécies, procedências, famílias e clones podem oferecer uma oportunidade de se alterar características importantes na madeira, na busca de se produzir matéria-prima com qualidade adequada na manufatura de diferentes produtos. Logo, estudos de espécies, procedências e clones vêm ganhando importância tendo em vista a adequação destes aos seus diversos usos (GUIMARÃES JUNIOR et al., 2011).

Neste sentido, o objetivo deste trabalho foi avaliar as propriedades físicas e mecânicas de painéis OSB produzidos com madeiras de clones de Eucalyptus urophylla.

\section{Material e Métodos}

Neste estudo foram utilizadas árvores de 6 clones de $E$ ucalyptus urophylla, com idade de aproximadamente 8 anos, incluindo 5 árvores por clone. Os testes clonais foram instalados nas Fazendas Riacho e Bom Sucesso, de propriedade da Companhia Mineira de Metais - Unidade Agroflorestal (VMAGRO), localizadas no município de Vazante, região noroeste de Minas Gerais. A área está situada a 17³6’09" Sul de latitude, longitude de $46^{\circ} 42^{\prime} 42^{\prime \prime}$ Oeste de Greenwich e altitude de 550 metros. Segundo a classificação de Köppen, o clima é do tipo Aw, tropical úmido de savana, com inverno seco e verão chuvoso, com temperatura média anual de $24^{\circ} \mathrm{C}$ e precipitação média anual de $1.450 \mathrm{~mm}$.

Na Tabela 1 são apresentadas as características dos clones de Eucalyptus urophylla utilizados nessa pesquisa (GUIMARÃES JUNIOR et al., 2012). A densidade básica foi obtida pela divisão da massa seca pelo volume saturado dos corpos de prova.

Tabela 1. Valores médios de diâmetro à $1,30 \mathrm{~m}$ de altura e densidade básica, obtida por média ponderada, dos clones de Eucalyptus urophylla.

Table 1. Mean values of diameter and basic density of Eucalyptus urophylla clones.

\begin{tabular}{ccc}
\hline Clone & Diâmetro $(\mathrm{cm})$ & Densidade básica $\left(\mathrm{g} / \mathrm{cm}^{3}\right)$ \\
\hline 19 & 34,69 & 0,65 \\
26 & 34,37 & 0,60 \\
28 & 32,15 & 0,52 \\
36 & 37,24 & 0,59 \\
58 & 32,91 & 0,56 \\
62 & 26,10 & 0,53 \\
\hline
\end{tabular}


As árvores foram seccionadas em toras, as quais foram cortadas em toretes de 1 metro e, para a produção das partículas "strand", utilizou-se os toretes da parte basal das árvores. Para tanto, estes foram desdobrados em tábuas, com aproximadamente $80 \mathrm{~cm}$ de comprimento, $12 \mathrm{~cm}$ de largura e 2,0 cm de espessura. Estas foram reduzidas a peças com 20 cm de comprimento, sendo que a largura e espessura permaneceram as mesmas. Após isso, foram armazenadas em tanques com água e submergidas até a ocorrer a saturação, para facilitar o processo posterior de corte.

Em seguida, as peças foram processadas em um picador, gerando partículas do tipo "strand" com comprimento de 85 $\mathrm{mm}, 25 \mathrm{~mm}$ de largura e espessura de 0,60 $\mathrm{mm}$. Estas foram posteriormente secas em estufa com circulação forçada de ar até $4 \%$ de umidade na base seca e peneiradas para a retirada de partículas menores.

Foi utilizado o adesivo fenol-formaldeído, na proporção de $9 \%$ com base na massa seca do painel, o qual apresentava teor de sólidos de 50,87\%; pH de 12,45; viscosidade de 595Cp e Gel Time de 10,12 minutos. Também foi aplicada a emulsão de parafina, na proporção de $1 \%$. Após a formação do painel o mesmo passou pelo processo de pré-prensagem, em prensa manual, com aplicação de pressão de $0,78 \mathrm{MPa}$ por 8 minutos.

O ciclo de prensagem utilizado incluiu temperatura de $150^{\circ} \mathrm{C}$, pressão específica de 3,92 MPa e tempo de prensagem de 10 minutos. Foram produzidos 6 painéis para cada clone avaliado, totalizando 36 painéis. As dimensões e a densidade nominal foram, respectivamente, de $480 \mathrm{~mm}$ x $480 \mathrm{~mm}$ x 15 $\mathrm{mm}$ e $0,70 \mathrm{~g} / \mathrm{cm}^{3}$. O colchão de partículas foi montado de forma orientada e foi composto por três camadas cruzadas, na proporção de 20:60:20 (capa, miolo, capa).

Para avaliação das propriedades físicas e mecânicas, foram retirados, de cada painel, quatro corpos de prova para o teste de tração perpendicular, quatro para densidade, umidade, absorção de água e inchamento em espessura e dois para flexão estática, sendo que neste último determinaram-se os módulos de elasticidade e de ruptura (MOE e MOR, respectivamente). Os mesmos foram climatizados à temperatura de $20 \pm 3^{\circ} \mathrm{C}$ e umidade relativa de $65 \pm 5 \%$ até a estabilização de suas respectivas massas. Os ensaios foram realizados de acordo com os procedimentos descritos nas normas EN 319, EN 317 e EN 310 , respectivamente.

Os resultados foram analisados estatisticamente por meio de ANOVA e teste de Scott-Knott em nível de 95\% de confiança.

\section{Resultados e Discussão}

Todos os painéis produzidos apresentaram valores de densidade aparente iguais estatisticamente, os quais variaram entre 0,65 e $0,69 \mathrm{~g} / \mathrm{cm}^{3}$ (Tabela 2 ).

Tabela 2. Valores médios de densidade aparente e razão de compactação dos painéis OSB de clones de Eucalyptus urophylla.

Table 2. Mean values of bulk density and compression ratio of OSB panels Eucalyptus urophylla clones.

\begin{tabular}{cccc}
\hline Clone & $\begin{array}{c}\text { Densidade aparente } \\
\left(\mathrm{g} / \mathrm{cm}^{3}\right)\end{array}$ & $\begin{array}{c}\text { Razão de } \\
\text { compactação }\end{array}$ & $\begin{array}{c}\text { Umidade } \\
(\%)\end{array}$ \\
\hline 19 & $0,65 \mathrm{~A}^{*}$ & $1,10 \mathrm{~A}$ & $8,12 \mathrm{~A}$ \\
26 & $0,67 \mathrm{~A}$ & $1,11 \mathrm{~A}$ & $8,45 \mathrm{~A}$ \\
28 & $0,69 \mathrm{~A}$ & $1,37 \mathrm{C}$ & $8,47 \mathrm{~A}$ \\
36 & $0,68 \mathrm{~A}$ & $1,14 \mathrm{~A}$ & $8,82 \mathrm{~A}$ \\
58 & $0,69 \mathrm{~A}$ & $1,23 \mathrm{~B}$ & $7,98 \mathrm{~A}$ \\
62 & $0,66 \mathrm{~A}$ & $1,24 \mathrm{~B}$ & $7,75 \mathrm{~A}$ \\
\hline $\mathrm{CV}(\%)$ & 7,87 & 8,01 & 10,32
\end{tabular}

${ }^{\star}$ Médias seguidas de mesma letra nas colunas não diferem entre si pelo teste de Scott-Knott a 5\% de significância.

Com relação às densidades médias obtidas para os painéis, estas foram ligeiramente inferiores em comparação à densidade nominal de $0,70 \mathrm{~g} / \mathrm{cm}^{3}$, estabelecida no delineamento 
experimental. Tais diferenças podem ser atribuídas, sobretudo, às condições operacionais relacionadas à perda de materiais durante a formação do colchão e prensagem dos painéis, além do retorno em espessura do painel após a sua retirada da prensa. Esses fatores influenciam diretamente na redução da densidade do painel em função da redução da massa de partículas do colchão e aumento da espessura do painel.

Ao avaliar o desempenho de painéis OSB, Carvalho et al. (2015), observaram a mesma tendência desde trabalho em relação à densidade dos painéis, que se mostrou inferior aquela determinada inicialmente de $0,65 \mathrm{~g} / \mathrm{cm}^{3}$, em que obtiveram valores variando entre $0,54 \mathrm{e} 0,57 \mathrm{~g} / \mathrm{cm}^{3}$.

Em trabalho realizado por Gorski et al. (2015), no qual os autores avaliaram a produção de painéis OSB com partículas de Eucalyptus benthamii, foi observado aumento da espessura pré-determinada dos painéis, na ordem de até $1,86 \mathrm{~mm}$. Os autores atribuíram esse comportamento ao grande volume de partículas no colchão para atingir a densidade nominal, o que tem como consequência uma maior liberação parcial das tensões de compressão após o final da prensagem na fase de produção dos painéis.

Os painéis OSB produzidos com os clones de eucalipto foram classificados como de média densidade, por apresentarem valores para essa propriedade entre 0,59 a $0,80 \mathrm{~g} / \mathrm{cm}^{3}$, de acordo com classificação sugerida por Iwakiri (2005).

Para a razão de compactação apenas o clone 28 apresentou valor dentro da faixa ideal $(1,37)$, segundo a classificação de Maloney (1993), que indica valores para esta propriedade entre 1,3 e 1,6. Isso se deve ao fato de esse clone apresentar menor densidade da madeira em relação aos demais, já que a razão de compactação segue tendência decrescente de acordo com o aumento da densidade da matéria-prima empregada, mantendo-se a densidade aparente dos painéis produzidos. Ainda de acordo com Maloney (1993), painéis com alta razão de compactação apresentam maior área de contato entre as partículas, gerando influências positivas nas propriedades mecânicas.

Não foram observadas diferenças entre os tratamentos com relação a umidade, o quais apresentaram valor médio de $8,27 \%$. Nota-se que estes atenderam aos requisitos estipulados pela norma NBR 14.810 (2006) que estabelece valores de teor de umidade entre $5 \%$ e $11 \%$.

Nas Figuras 1 e 2 estão apresentados os comportamentos de absorção de água e inchamento em espessura após 2 e 24 horas de imersão.

Para a absorção de água após 2 horas de imersão, os clones 26 e 62 apresentaram valores superiores aos demais, sendo estes de 30,41 e 33,47\%, respectivamente. Já para a absorção após 24 horas de imersão não se observou diferenças entre os clones, sendo que os valores variaram entre 49,58 e 62,94\%.

Ao avaliar chapas de partículas "strand" de duas espécies nativas da mata atlântica, Vidaurre et al. (2004) obtiveram entre 21 a 33\% e 45 a 58\% de absorção de água após 2 e 24 horas de imersão, respectivamente, valores estes correspondentes com os observados neste trabalho.

O clone 62 mostrou maior inchamento em espessura após 2 horas de imersão, com média de 9,43\%. Os demais materiais genéticos apresentaram valores estatisticamente iguais e inferiores ao clone 62. Após 24 horas de imersão, o clone 28 foi o que apresentou maior inchamento, com 28,14\%.

Em trabalho realizado com painéis OSB de Eucalyptus grandis com $8 \%$ de adesivo fenol-formaldeído, Okino et al. (2008), observaram inchamento em espessura após 2 e 24 horas de imersão de 14,6 e 26,8\%, respectivamente. Nota-se, portanto, que os valores de inchamento em espessura observados se mostraram próximos aos referenciados em literatura.

A CSA 0437-0 (1993) estabelece valor máximo de 10\% para inchamento em espessura após 24 horas de imersão em água. Neste sentido, os clones apresentaram valores acima do 
limite máximo estipulado pela norma. Esse fenômeno pode ter ocorrido devido aos valores de razão de compactação serem baixos. Para todas as propriedades físicas, a diminuição da razão de compactação reduz o desempenho dos painéis aglomerados devido à menor barreira física à entrada de água causada pelo aumento dos espaços vazios entre as partículas

(BUFALINO et al., 2012).

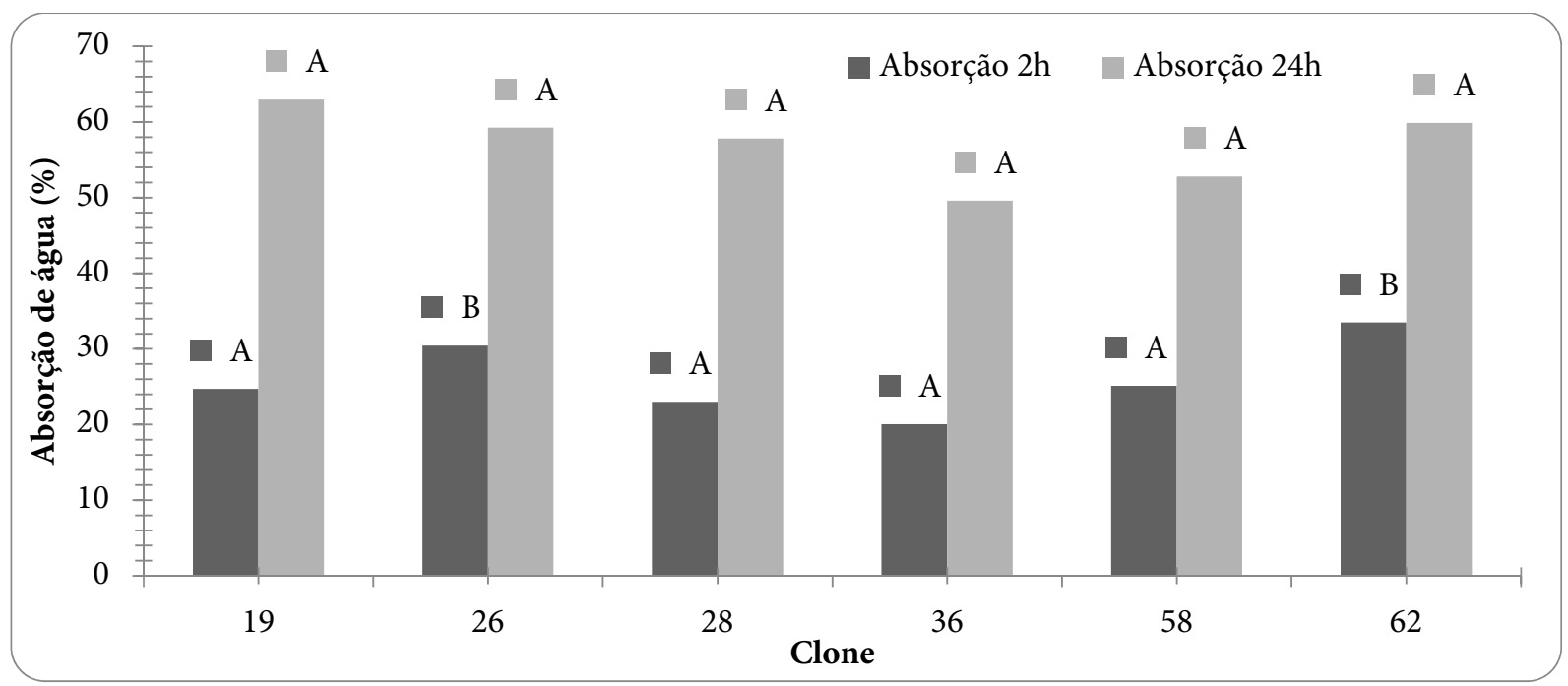

Figura 1 - Absorção de água em 2 e 24 horas de imersão em água.

Figure 1 - Water absorption at 2 and 24 hours of immersion in water.

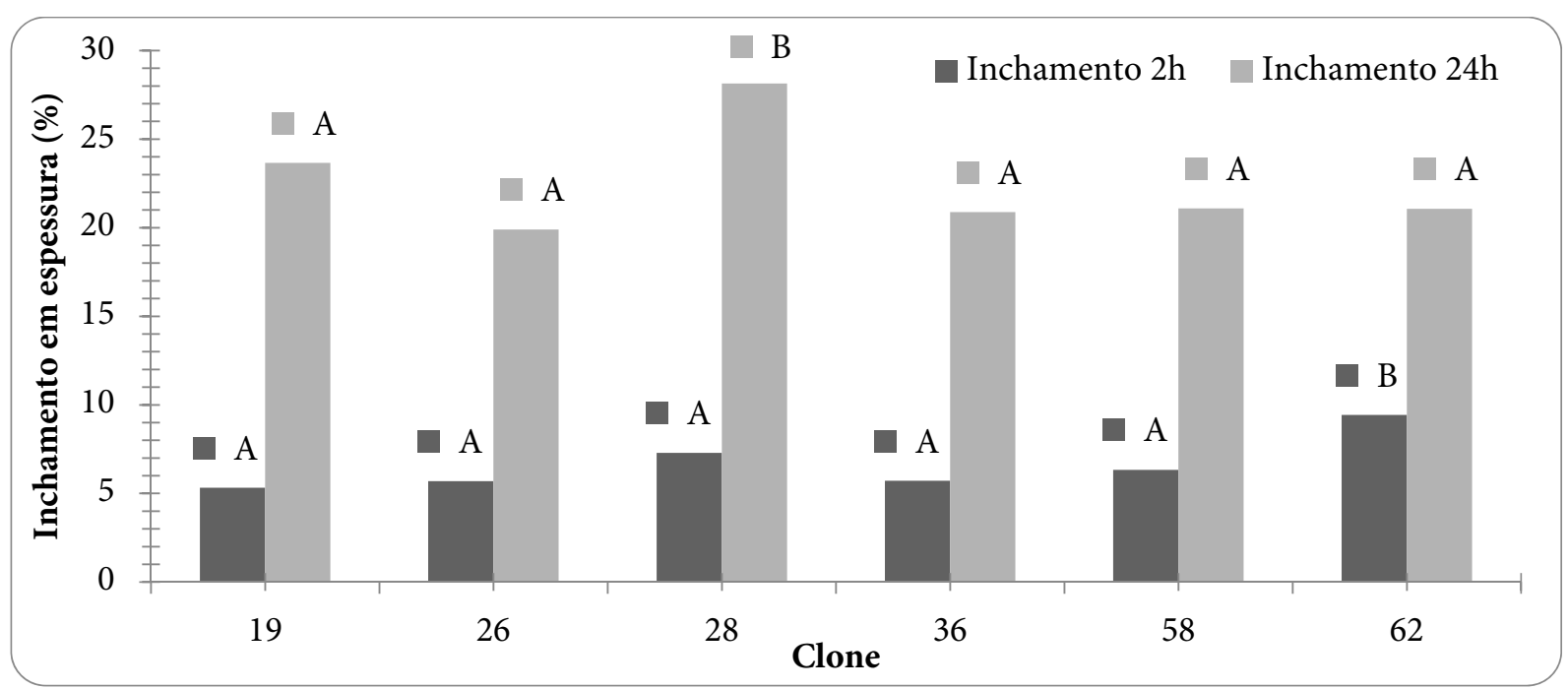

Figura 2 - Inchamento em espessura em 2 e 24 horas de imersão em água.

Figure 2 - swelling in thickness in 2 and 24 hours immersion in water.

Na Tabela 4 estão os valores observados para as propriedades mecânicas dos painéis OSB produzidos com as madeiras de clones de Eucalyptus urophylla.

O clone 19 foi o que apresentou melhor comportamento no teste de flexão estática, tanto para MOE quanto para MOR avaliados no sentido paralelo. Quando essas propriedades foram avaliadas no sentido perpendicular, todos os clones apresentaram resultados estatisticamente iguais, com valores médios MOE e MOR de 1678,14 e 15,08 MPa, respectivamente. 
Tabela 4. Valores médios de módulo de ruptura (MOR), módulo de elasticidade (MOE) e tração perpendicular dos painéis OSB avaliados.

Table 4. Mean values for modulus of rupture (MOR), modulus of elasticity (MOE) and perpendicular traction of assessed OSB.

\begin{tabular}{|c|c|c|c|c|c|}
\hline Clones & $\begin{array}{c}\text { MOE } \\
\text { paralelo }\end{array}$ & $\begin{array}{c}\text { MOR } \\
\text { paralelo }\end{array}$ & $\begin{array}{c}\text { MOE } \\
\text { perpendicular }\end{array}$ & $\begin{array}{c}\text { MOR } \\
\text { perpendicular }\end{array}$ & $\begin{array}{c}\text { Tração } \\
\text { perpendicular }\end{array}$ \\
\hline & $\mathrm{MPa}$ & $\mathrm{MPa}$ & $\mathrm{MPa}$ & $\mathrm{MPa}$ & $\mathrm{MPa}$ \\
\hline 19 & $6651,62 \mathrm{~B}^{*}$ & $48,60 \mathrm{~B}$ & $1658,32 \mathrm{~A}$ & $18,56 \mathrm{~A}$ & $0,40 \mathrm{~A}$ \\
\hline 26 & $3307,56 \mathrm{~A}$ & $30,80 \mathrm{~A}$ & $1667,46 \mathrm{~A}$ & $15,32 \mathrm{~A}$ & $0,31 \mathrm{~A}$ \\
\hline 28 & $5075,61 \mathrm{~A}$ & $29,44 \mathrm{~A}$ & $1543,71 \mathrm{~A}$ & $13,61 \mathrm{~A}$ & $0,33 \mathrm{~A}$ \\
\hline 36 & $3994,72 \mathrm{~A}$ & $30,38 \mathrm{~A}$ & $2016,51 \mathrm{~A}$ & $16,16 \mathrm{~A}$ & $0,38 \mathrm{~A}$ \\
\hline 58 & $4181,78 \mathrm{~A}$ & $34,20 \mathrm{~A}$ & $1685,91 \mathrm{~A}$ & $13,21 \mathrm{~A}$ & $0,35 \mathrm{~A}$ \\
\hline 62 & $4482,15 \mathrm{~A}$ & $39,29 \mathrm{~A}$ & $1496,94 \mathrm{~A}$ & $13,64 \mathrm{~A}$ & $0,30 \mathrm{~A}$ \\
\hline CV(\%) & 36,30 & 31,46 & 35,05 & 15,23 & 46,29 \\
\hline
\end{tabular}

${ }^{\star}$ Médias seguidas de mesma letra nas colunas não diferem entre si pelo teste de Scott-Knott a 5\% de significância.

Para painéis OSB produzidos com madeira de Cupressus glauca, Okino et al. (2007), encontraram valores de MOE de 4.667 e $2.524 \mathrm{MPa}$ e para MOR na ordem de 50,9 e 34,6 MPa, avaliados nos sentidos paralelo e perpendicular, respectivamente.

Para MOR e MOE avaliados no sentido paralelo, a norma CSA 0437 (1993), determina valores mínimos de 29,1 e 5510,7 $\mathrm{MPa}$, respectivamente, para painéis OSB classe O-2. Sendo assim, para o MOE apenas o clone 19 apresentou valor que satisfaz a norma. Para o MOR, todos os clones apresentaram valores acima do mínimo estipulado. A mesma norma determina, para avaliação no sentido perpendicular, valores mínimos 12,4 MPa para o MOR e 1502,9 MPa para o MOE. Dessa forma, todos os clones apresentaram resultados de acordo com as exigências normativas, com exceção do clone 62, que apresentou valor levemente abaixo do mínimo estabelecido, para o MOE perpendicular.

Todos os clones avaliados apresentaram valores de tração perpendicular estatisticamente iguais, o que pode ser atribuído aos valores similares de densidade dos painéis, não demonstrando influência da razão de compactação para essa propriedade. Apenas os clones 19, 36 e 58 apresentaram valores acima do mínimo estipulado pela norma CSA 0437, de $0,34 \mathrm{MPa}$.

Ao estudarem diversas espécies de eucalipto na produção de painéis OSB em comparação com Pinus taeda, Iwakiri et al. (2004), obtiveram valores de tração perpendicular entre 0,11 e 0,27 MPa, sendo esses valores inferiores aos observados nesse trabalho, o que pode ser explicado pela utilização de madeiras de maior densidade e, consequentemente menores valores de razão de compactação.

\section{Conclusões}

Houve baixo efeito clonal nas propriedades físicas e mecânicas dos painéis OSB. O clone 19 se destacou com os melhores resultados em todas as propriedades avaliadas. Para as propriedades físicas, nenhum dos tratamentos atendeu a normatização com relação ao inchamento em espessura após 24 horas. Nas propriedades mecânicas se adequaram às exigências normativas: tração perpendicular os clones 19, 36 e 58; MOE paralelo o clone 19; MOE perpendicular todos os clones com exceção do 62; MOR paralelo e perpendicular todos os clones. 


\section{Referências}

BERTOLINI, M. S., et al. Accelerated artificial aging of particleboards from residues of CCB treated Pinus sp. and castor oil resin. Materials Research, v. 16, n. 2, p. 293-303, 2013. BUFALINO, L. et al. Modelagem das propriedades físicas e mecânicas em painéis aglomerados de cedro australiano. Floresta e ambiente, Rio de Janeiro, v. 19, n. 2, p. 243-249, 2012.

CANADIAN STANDARDS ASSOCIATION. OSB and Waferboard. CSA 0437.0 - 93. Ontário: 1993. 18p.

CARVAlHO, A. G, et al. Desempenho de painéis OSB com adesivos comerciais e tânico de barbatimão. Revista Árvore, Viçosa, v.39, n.6, p. 1155-1163, 2015.

CASTRO, M. B. Resistência mecânica de painéis OSB de pinus e eucalipto. In: ENCONTRO BRASILEIRO EM MADEIRAS E EM ESTRUTURAS DE MADEIRA, 13., 2012, Vitória. Anais... Vitória: UFES, 2012.

EUROPEAN COMMITTEE FOR STANDARDIZATION. CEN 02.26. 1993. EN 310: wood-based panels; EN 317: particleboards and fiberboards; EN 319: particleboards and fiberboards. Brussels, 1993.

GORSKI, L., et al. Utilização da madeira DE Eucalyptus benthamii na produção de painéis de partículas orientadas (OSB). Floresta, Curitiba, v. 45, n.4, p. 865-874, 2015.

GUIMARÃES JÚNIOR, et al. Painéis de madeira aglomerada de resíduos da laminação de diferentes procedências de $E u$ calyptus grandis, Eucalyptus saligna e Eucalyptus cloeziana. Cerne, Lavras, v.17, n.4, p.443-452, 2011.

GUIMARÃES JÚNIOR, J. B., et al. Seleção de clones de Eucalyptus urophylla para produção de compensados. Cerne, Lavras, v.18, n.4, p.667-673. 2012.

IWAKIRI, S.; et al. Utilização da madeira de eucalipto na produção de chapas de partículas orientadas - OSB. Cerne, Lavras, v. 10, n. 1, p. 46 - 52, 2004.

IWAKIRI, S. Painéis de madeira reconstituída. Curitiba: FUPEF, 2005. 247 p.

MALONEY, T. M. Modern particleboard and dry-process fiber board manufacture. San Francisco: Miller Freeman. 1993. $689 \mathrm{p}$.

MENDES, R.F., et al. Effect of thermal treatment on properties of OSB panels. MENDES, Rafael Farinassi et al. Effect of thermal treatment on properties of OSB panels. Wood science and technology, v. 47, n. 2, p. 243-256, 2013.

MARTARELLO, L. P., et al.Influência do arranjo das camadas do colchão nas propriedades tecnológicas de painéis de partículas orientadas (OSB) de Eucalyptus benthamii. Scientia Forestalis, v. 43, n. 107, p.503-510. 2015.

OKINO, E. Y.; TEIXEIRA, D. E.; DEL MENEZZI, C. H. Postthermal treatment of oriented strandboard (OSB) made from cypress (Cupressus glauca Lam.). Maderas. Ciencia y tecnología, v. 9, n. 3, p. 199-210, 2007.

OKINO, E. Y. A. et al. Propriedades de Chapas OSB de Eucalyptus grandis e de Cupressus glauca. Scientia Forestalis, Piracicaba, v. 36, n. 78, p. 123-131, 2008.

VIDAURRE, G. B. et al. Produção de chapas de partículas de madeira de duas espécies nativas da Mata Atlântica e suas combinações. Ciência Florestal, Santa Maria, v. 14, n. 1, p. 235-242, 2004. 